\title{
Les antidépresseurs de la performance
}

\section{Performance antidepressant drugs}

Jean-Pierre GOULLÉ*, Christian LACROIX

Laboratoire de Pharmacocinétique et de Toxicologie Clinique, Groupe Hospitalier BP 24 - 76083 LE HAVRE Cedex - Tél : 0232733218 - Fax : 0232733238

*Auteur à qui adresser la correspondance : Docteur Jean-Pierre GOULLÉ, Laboratoire de Pharmacocinétique et de Toxicologie Clinique, Groupe Hospitalier, Jacques Monod - BP 24 76083 LE HAVRE Tél : 0232733223 - Fax : 0232733238

(Reçu le 17 décembre 1999 ; accepté le 15 janvier 2000)

\section{RÉSUMÉ}

En France, au cours de la période 1991-1997, alors que la vente des médicaments psychotropes a augmenté de $7 \%$, le marché des antidépresseurs s'est littéralement envolé avec une progression de plus de $40 \%$. Cette consommation, 2 à 3 fois plus importante que celle de nos voisins européens, est génératrice d'abus conduisant à une accoutumance, à une assuétude, voire à une véritable conduite toxicophile. Les antidépresseurs $(A D)$ font partie des substances qui sont utilisées par l'homme pour améliorer sa performance, en raison de leurs effets pharmacologiques. Les études chez l'animal montrent qu'un certain nombre d'AD ont des propriétés stimulantes. Très vite, ces molécules sont détournées de leur usage à des fins de dopage, parfois avant même d'être utilisées en thérapeutique. L'exemple le plus récent est celui du bromantan Russe, agent à la fois psychostimulant et masquant. Si les observations de pharmacodépendance aux $A D$ tricycliques sont rares et anecdotiques, il en va différemment avec les nouveaux antidépresseurs : inhibiteurs de recapture des amines pressives en particulier. Ces produits sont dénués de toxicité cardiaque, malheureusement de nombreux autres effets secondaires ou pervers voire toxiques sont apparus à l'usage. Pour des raisons de santé publique une demi douzaine d'AD ont été retirés du marché au cours des dernières années. Ainsi l'amineptine a fait l'objet d'un retrait en France (30/06/99) tant les observations de surconsommation étaient nombreuses. D'autres $A D$ font l'objet d'un détournement d'usage et sont passés en revue. Il est souligné que l'analyse des cheveux s'avère un complément extrêmement utile dans la recherche de ces médicaments.

MOTS-CLÉS

Antidépresseurs, performance.

\section{SUMMARY}

Whereas in France the sale of psychotropic medication increased $7 \%$ in the period between 1991 and 1997, the antidepressant drugs market literally exploded with a progression of $40 \%$. This consumption 2 to 3 times greater than that of our European neighbours created an abuse which lead to habituation, to an addiction, and even to drug abuse. Antidepressant drugs (AD) pertain to the substances which are used by man to improve its performance due to its pharmacological effects. Animal studies have shown that a certain number of $A D$ have stimulating properties. These molecules are very soon diverted from their intended use to be utilized with doping purposes, sometimes even before being therapeuticaly employed. The most recent example is that of Russian bromantan, at the same time a psychostimulant and a masking agent. If the observations of pharmacodependency on tricyclical $A D$ are rare and anecdotal we cannot say the same for the new antidepressant drugs, in particular pressive amines recapture inhibitors. These products are devoid of cardiac toxicity, unfortunately however, a series of other secondary even toxic effects have become evident after usage. Because of public health reasons half a dozen antidepressant drugs were retired from the market in the last years. Amineptine was thus retired from the French market (30/06/99) because of the numerous observations of overconsumption. Other $A D$ are also being used otherwise and are thus being reviewed. It is underlined that hair analysis provides a very useful tool in the search of these medications.

\section{KEY-WORDS}

Antidepressant drugs, performance. 


\section{Introduction}

Les antidépresseurs (AD) font partie des molécules qui sont utilisées par l'homme pour améliorer sa performance physique. Ainsi dans le dopage d'état organisé par les pays de l'est il y a quelques années, les $\mathrm{AD}$ font partie de la panoplie du sportif : "la décision de doper venait d'en haut. Un rapport de la stasi fait état de la décision d'explorer diverses procédures : prise d'anabolisants, d'antidépresseurs, et l'électrostimulation" (1). La profonde mutation du mode de vie au cours du $20^{c}$ siècle avec sa cohorte d'effets pervers : pression, stress, insomnie est à l'origine de l'usage massif des AD. La fluoxétine (Prozac $\left.{ }^{\oplus}\right)$, médicament qui permet de se sentir "mieux que bien" comme disent les Américains, est consommé chaque jour par 30 millions de personnes dans le monde. C'est le $3^{\mathrm{c}}$ médicament le plus vendu, avec un chiffre d'affaires annuel de 12 milliards de francs. En France, au cours de la période 1991-1997 si la prescription de psychotropes a progressé de $7 \%$, au sein de cette classe les AD ont connu un véritable boum avec $+42,5 \%$ ! Cette situation est inquiétante puisque cette consommation est 2 à 3 fois supérieure à nos voisins Européens, 3 fois plus élevée qu'en Allemagne. Outre les véritables indications médicales, il existe un détournement d'usage manifeste. Ainsi un cadre sur cinq est dopé (2). Les médecins du travail notent que le mal sévit particulièrement dans les secteurs à fortes tensions où prime la performance individuelle : cabinets de conseil, grands réseaux de distribution, finance, banque, informatique (2).

\section{Rappel concernant les anti- dépresseurs (AD)}

Le déterminant essentiel de la dépression est un déficit en amines pressives. Les $\mathrm{AD}$ exerceraient leur action en augmentant le temps de séjour de ces amines au niveau synaptique soit par inhibition de leur recapture, soit inhibition de leur dégradation (3).

\section{Antidépresseurs inhibiteurs non sélectifs de recapture (IR) tricycliques, tétracy- cliques}

Leur mécanisme d'action est une inhibition de recapture de la nor-adrénaline (IRNAd), de la sérotonine et pour certains de la dopamine.

\section{Antidépresseurs inhibiteurs de la Mono Amine Oxydase (IMAO)}

\section{Non sélectifs (inhibiteurs irréversibles)}

Leur mécanisme d'action est voisin des précédents. Il s'agit d'antidépresseurs tricycliques (ADT) et tétracycliques (ce sont des inhibiteurs de recapture de la NAd, sérotonine, dopamine) avec effet euphorisant marqué. En raison d'effets secondaires et d'interactions marqués leur utilisation est exceptionnelle dans le cadre qui nous intéresse.

\section{Sélectifs}

Seuls les IMAO inhibiteurs réversibles de type A exercent un effet antidépresseur : Toloxatone (Humory ${ }^{\circledR}$ ) et Moclobémide (Moclamine ${ }^{\circledR}$ ) appartiennent à cette catégorie.

\section{Antidépresseurs inhibiteurs de recaptu- re de la Sérotonine (IRS) et de la nor- adrénaline (IRNAd)}

Il s’agit du Milnacipran $\left(\right.$ Ixel $\left.^{\circledR}\right)$, Venlafaxine (Effoxor ${ }^{\circledR}$ ).

\section{Antidépresseurs inhibiteurs de recaptu- re spécifique à la Sérotonine (IRSS).}

Ces molécules présentent un index de sélectivité sérotoninergique important, surtout si on le compare aux ADT où il est inférieur à 1 (Tableau I).

Leur demie-vie est voisine de 24 heures, à l'exception de la fluoxétine qui est de 48 à 72 heures.

Tableau I : Index de sélectivité sérotoninergique des nouveaux antidépresseurs.

\begin{tabular}{|l|l|}
\hline $\begin{array}{l}\text { Index de sélectivité } \\
\text { sérotoninergique }\end{array}$ & \\
\hline 1000 à 10000 & Séropram $\left(\right.$ Citalopram $\left.^{\circledR}\right)$ \\
\hline 100 à 1000 & $\begin{array}{l}\text { Sertraline }\left(\text { Zoloft }^{\oplus}\right) \\
\text { Paroxetine }(\text { Deroxat }\end{array}$ \\
& Fluvoxamine $\left(\right.$ Floxyfral $\left.^{\oplus}\right)$ \\
\hline 10 à 100 & Fluoxétine $\left(\right.$ Prozac $\left.^{\circledR}\right)$ \\
\hline
\end{tabular}

\section{Antidépresseurs inhibiteurs de recaptu- re spécifique de la Dopamine (IRSDo)}

Cette classe est représentée par l'Amineptine (Survector ${ }^{\circledast}$ ), molécule chimiquement tricyclique mais qui, du fait de son mode d'action (IRSDo), possède à la fois des propriétés antidépressives et stimulantes.

\section{Antidépresseurs stimulants de recapture de la Sérotonine (SRS)}

Le seul représentant est la Tianeptine (Stablon $\left.{ }^{\circledR}\right)$. 


\section{Effets indésirables et phar- macodépendance des AD}

Deux molécules présentent un risque très élevé ou élevé de pharmacodépendance : respectivement $79 \%$ et $21,5 \%$ pour l'amineptine et pour la tianeptine (4). En ce qui concerne la fluoxétine, il s'agit plutôt d'une dépendance psychique (5). Les observations de pharmacodépendance aux ADT sont rares et anecdotiques (6) vraisemblablement en raison de la toxicité myocardique de ces molécules. Par ailleurs chez l'homme, l'amitriptyline semble réduire la puissance musculaire cinétique (7). En revanche, des syndromes de sevrage ont été fréquemment constatés (8). L'incidence évaluée à partir d'études rétrospectives ou de cas uniques varie entre 21 et $55 \%$. Des consommations abusives ont été décrites pour l'amitriptyline (Laroxyl ${ }^{\circledR}$ ), la trimipramine $\left(\right.$ Surmonti $\left.{ }^{\circledR}\right)$, la tianeptine $\left(\right.$ Stablon $^{\circledR}$ ) et l'amineptine (Survector ${ }^{\circledR}$ ). Les principaux effets indésirables des AD sont reportés dans le tableau II.

\section{L'usage détourné des AD Amineptine (Survector $\left.{ }^{(}\right)$}

\section{- Historique}

L'autorisation de mise sur le marché (AMM) date de 1978 et dès 1983 des observations de surconsommation sont rapportées. Il faudra attendre 11 ans pour interdire toute publicité à destination des médecins, 15 ans pour que cette molécule soit classée comme substance dopante (J.O. 17/6/98) et 16 ans pour que l'AMM soit suspendue (30/1/99 et 30/6/99).

Tableau II : Pourcentage des principaux effets indésirables psychiatriques selon Blayac et coll (4).

\section{- Surconsommation d'amineptine}

Le résultat d'une étude multicentrique des centres français de pharmacovigilance (9) révèle que la surconsommation moyenne est de $2450 \mathrm{mg} / \mathrm{J}$ (600 à $12000 \mathrm{mg}$ 6 à $120 \mathrm{cp}$ ) pour posologie maximale recommandée de 100 à $200 \mathrm{mg} / \mathrm{J}$ soit 1 à $2 \mathrm{cp}$. Les prises sont fractionnées, en effet, la courte durée de l'effet psychostimulant euphorisant et dynamisant amène à une prise toutes les 2 heures. Il s'agit d'une véritable boulimie amenant à consommer plusieurs dizaines de comprimés chez le sportif au moment de l'effort et chez le cadre pendant la journée de travail. Ces surconsommations sont le plus souvent induites initialement par une prescription à des fins thérapeutiques, au moins chez le cadre. La surconsommation est généralement bien tolérée, si l'on considère les posologies parfois monstrueuses (jusqu'à 60 fois la posologie maximale recommandée) (5). Les effets indésirables sont neurologiques (agitation, confusion ) et dermatologiques : acné $(10,11)$. Le sevrage des sujets dépendants est particulièrement difficile à réaliser compte tenu de la dépendance psychique sévère et le plus souvent d'un terrain dépressif. Seulement $30 \%$ des patients sont abstinents après un mois de sevrage (12). Suite à sa détection dans plusieurs échantillons, cyclistes en majorité, par des laboratoires du Comité International Olympique (13), cette substance a été interdite en pratique sportive. L'approvisionnement fait appel aux moyens habituels des situations de pharmacodépendance (recours à plusieurs prescripteurs, fabrication d'ordonnances, vol, marché parallèle, internet). L'absence d'amélioration significative a conduit l'agence du médicament à interdire dans un premier temps la publicité médicale (14) puis au retrait de 1'AMM (15). A l'heure actuelle, il est très facile de se procurer ce médicament par internet : V. Dumestre, comment se doper via internet in "Les substances de la performance chez le sportif, le cadre et l'animal" Forum Labo, mars 2000, Paris.

\begin{tabular}{|lccccccccc|}
\hline & $\begin{array}{c}\text { Confusion } \\
\text { mentale }\end{array}$ & $\begin{array}{r}\text { Somnolence } \\
\text { Hallucinations }\end{array}$ & $\begin{array}{c}\text { Réaction } \\
\text { Maniaque }\end{array}$ & Angoisse & $\begin{array}{c}\text { Tentative } \\
\text { de suicide }\end{array}$ & Suicide & $\begin{array}{c}\text { Syndrome } \\
\text { de sevrage }\end{array}$ & Divers \\
\hline $\begin{array}{l}\text { Fluoxétine } \\
n=198\end{array}$ & $34 \%$ & $11,5 \%$ & $11,5 \%$ & $6,5 \%$ & $4 \%$ & $5,5 \%$ & $6,5 \%$ & $1,5 \%$ & $19 \%$ \\
$\begin{array}{l}\text { Fluvoxamine } \\
n=63\end{array}$ & $39 \%$ & $9,5 \%$ & $8 \%$ & $5 \%$ & $9,5 \%$ & $4,8 \%$ & $0 \%$ & $13 \%$ & $11,2 \%$ \\
$\begin{array}{l}\text { Paroxétine } \\
n=43\end{array}$ & $32,5 \%$ & $14 \%$ & $14 \%$ & $4,5 \%$ & $2,3 \%$ & $4,7 \%$ & $0 \%$ & $14 \%$ & $14 \%$ \\
$\begin{array}{l}\text { Amineptine } \\
n=253\end{array}$ & $8 \%$ & $3 \%$ & $3 \%$ & $0,5 \%$ & $1,2 \%$ & $0,8 \%$ & $0,5 \%$ & $79 \%$ & $4 \%$ \\
$\begin{array}{l}\text { Miansérine } \\
n=80\end{array}$ & $26 \%$ & $20 \%$ & $12,5 \%$ & $1 \%$ & $5 \%$ & $1 \%$ & $0 \%$ & $2,5 \%$ & $32 \%$ \\
$\begin{array}{l}\text { Viloxazine } \\
n=65\end{array}$ & $55 \%$ & $12,5 \%$ & $12,5 \%$ & $1,5 \%$ & $0 \%$ & $1,5 \%$ & $0 \%$ & $1,5 \%$ & $15,5 \%$ \\
$\begin{array}{l}\text { Tianeptine } \\
n=42\end{array}$ & $26 \%$ & $14 \%$ & $5 \%$ & $2,5 \%$ & $5 \%$ & $0 \%$ & $0 \%$ & $21,5 \%$ & $26 \%$ \\
\hline
\end{tabular}




\section{- Amineptine et cheveux}

La présence d'amineptine dans les cheveux est un marqueur extrêmement précieux d'une exposition à l'amineptine comme le démontre l'observation suivante (16) : un homme de 45 ans est admis en dermatologie avec des lésions d'acné extrêmement sévères. L'analyse sanguine réalisée au $10^{\text {enc }}$ jour est négative. La mesure d'amineptine effectuée dans deux sections de cheveux révèle des concentrations de 12,23 et $8,06 \mathrm{ng} / \mathrm{mg}$. Après avoir donné les résultats au malade, ce dernier admet la consommation quotidienne de 60 comprimés de $100 \mathrm{mg}$ d'amineptine. Cet exemple illustre, si besoin est, l'intérêt de la détermination dans les cheveux d'un certain nombre de molécules dans le cadre du dopage.

\section{- L'amineptine chez l'animal}

Chez la souris, la stimulation dopaminergique mesurée par l'activité horizontale, l'activité verticale et le nombre de petits mouvements sans déplacement par l'amineptine est dose dépendante. Elle persiste 8 heures à la posologie de $20 \mathrm{mg} / \mathrm{kg}$, i.p. Les études physiologiques suggèrent un mécanisme d'action différent de celui exercé par la dexamphétamine $(17,18)$. Parmi les substances dopaminergiques, les études électrophysiologiques permettent de distinguer celles qui augmentent le relargage (amphétamine) ; augmentent la synthèse (levodopa) ; bloquent la recapture (cocaine, nomifensine, amineptine) (19). Chez le chien, l'amineptine améliore, de manière dose dépendante, la vigilance, l'attention, augmente la capacité d'attente, d'anticipation, d'apprentissage (20). Les techniques de microdialyse confirment l'augmentation de la dopamine extra-cellulaires dans différentes parties du cerveau (striatum, nucleus accumbens, cortex frontal) et une augmentation de la noradrénaline dans le cortex frontal et l'hippocampus dorsal (21).

\section{Tianeptine (Stablon ${ }^{\circledast}$ )}

Contrairement aux inhibiteurs de recapture de la sérotonime qui agissent au niveau postsynaptique, la tianeptine stimule sa recapture au niveau présynaptique et reste sans effet sur les mouvements de la noradrénaline et de la dopamine (22). Des cas de surconsommation de tianeptine ont été rapportés y compris chez le cadre. Un cas nancéien est décrit (23) : il s'agit d'un médecin de 35 ans, qui consommait 120 à 150 comprimés par jour (environ 9 par heure). Il avait auparavant une surconsommation d'amineptine. Une pharmacodépendance touche $21,5 \%$ des sujets traités (Tableau II).

\section{Fluoxétine (Prozac $\left.{ }^{\circledR}\right)$}

C'est le troisième médicament le plus vendu dans le monde : 30 millions de personnes traitées, 12 milliards de francs de chiffre d'affaires (1996). Cet inhibiteur de recapture de la sérotonine est doté de potentialités stimulantes en raison d'une action dopaminergique au niveau du cortex frontal, propriété que l'on retrouve avec les amphétamines, l'héroïne ou la cocaïne (24). De nombreux cas de dépendance physique et d'abus ont été décrits avec la fluoxétine (25-28). Il existe une toxicomanie à grande échelle à l'origine de troubles graves du comportement : bouffées délirantes, hallucinations, conduites agressives $(25,26)$. La fréquence des réactions maniaques est plus importante avec la fluoxétine qu'avec tous les autres nouveaux AD (Tableau II). Avec cette molécule, les syndromes de privation sont plus difficiles à mettre en évidence en raison de la demi-vie longue, mais la fréquence et l'intensité de ces problèmes sont plus grandes que ceux liés aux autres $\mathrm{AD}$ (29). Le syndrome de sevrage est aujourd'hui bien documenté par de nombreux auteurs (30). L'activité stimulante est recherchée par le cadre et le sportif mais cette activité est parfois difficile à canaliser puisque automutilation, suicides et meurtres seraient favorisés par la fluoxétine (Tableau II). La prévalence des tentatives de suicide est plus forte avec la fluoxétine qu'avec tous les autres nouveaux AD (Tableau II). Depuis 1997, la fluoxétine est utilisée par les marathoniens à 2 ou 3 fois la posologie usuelle en raison de l'euphorie qu'elle procure qui aide à supporter les longues distances (31).

\section{Les I.M.A.O.}

Ces médicaments ralentissent la métabolisation des amines pressives. Ils ont un effet psychotonique antalgique et vasodilatateur. Les sportifs les utilisent pour diminuer la tension nerveuse due au stress de la compétition. Ces molécules ne sont pas classées parmi les substances dopantes. Les syndromes de sevrage sont plus rares mais plus graves qu'avec les tricycliques (5). Les I.M.A.O. (en particulier les I.M.A.O. non sélectifs) sont de moins en moins prescrits pour ces raisons mais également à cause des interactions médicamenteuses et alimentaires. Nous ne disposons à l'heure actuelle d'aucune donnée en ce qui concerne leur usage chez le sportif. Pour les I.M.A.O. sélectifs de type A, des observations de prise toxicomaniaque ( 2 à 3 fois la posologie usuelle) de moclobémide (Moclamine ${ }^{\oplus}$ ) ont été rapportées en Finlande (32), à la recherche d'effets euphorisants.

\section{Autres molécules}

D'autres molécules font l'objet d'abus d'usage : paroxétine (Déroxat $\left.{ }^{\circledR}\right)$, sertraline (Zoloft $\left.{ }^{\boxplus}\right)$. Toutes deux sont des inhibiteurs sélectifs de recapture de la sérotonine pratiquement dépourvues d'effet direct sur la recapture de la noradrénaline, de la dopamine et de l'acide gamma-aminobutyrique. Des cas de syndromes de sevrage ont été décrits pour ces médicaments depuis 1993 pour la paroxétine et 1994 pour la sertraline (30,33-35). La fréquence des états délirants et des hallucinations est plus importan- 
te avec la paroxétine qu'avec tous les autres nouveaux $\mathrm{AD}$ (Tableau II). Par ailleurs sa (sur)consommation exercerait un effet négatif sur la capacité d'un individu à réaliser un exercice prolongé. En ce qui concerne la médifoxamine (Cledial $\left.{ }^{\Phi}\right)$, on note une activité sur les 3 systèmes monoaminergiques : noradrénaline, dopamine et sérotonine ce qui en fait un bon candidat potentiel, mais l'effet anticholinergique à doses élevées la rend peu propice à un détournement d'usage. Des syndromes de sevrage ont également été publiés avec la fluvoxamine (Floxyfral ${ }^{\circledR}$ ) depuis 1992 et la venlaflaxine (Effexor ${ }^{\circledR}$ ) depuis 1996 (30). La minaprine $\left(\right.$ Cantor $\left.^{\circledR}\right)$ est un psychostimulant doté de propriétés antidépressives atypiques. Ce médicament a été retiré du marché français en 1996 en raison de sa toxicité neurologique redoutable (Tableau III). La miansérine (Athymil ${ }^{\oplus}$ ), outre ses propriétés antidépressives possède une action anxiolytique et sédative et un effet régulateur du sommeil. Pour cette molécule comme pour la viloxazine (Vivalan ${ }^{\circledR}$ ) les effets indésirables psychiatriques sont assez nombreux (Tableau II). Le bromantan (dérivé p-bromophényl de la 2-amantadine) est une nouvelle substance psychoactive développée par l'institut de pharmacologie de Moscou il y a quelques années. Il s'agit d'un stimulant du système dopaminergique (Sérotonine, 5-hydroxyindoleacétique acide) doué d'effets psychostimulant mais également anxiolytique, immunostimulant et protecteur de membrane (36-40). Le bromantan a été reconnu comme produit dopant (41). Les premiers cas de positivité chez les sportifs ont été signalés en mars 1994 (13). Deux médaillés olympiques Russes (natation et lutte) ainsi que deux autres compétiteurs (une nageuse Russe, une cycliste Lithuanienne) ont été positifs au bromantan à Atlanta en 1996. Déchus de leur titre, ils ont été réintégrés après que le comité olympique Russe ait fait appel prétextant que cette substance ne figurait pas sur la liste ! Cette molécule a été ajoutée sur la liste des produits dopants en 1996. Le cycliste Abdujaparov a été exclu du tour de France 1997 après avoir été contrôlé positif au bromantan et au clembutérol.

\section{Conclusion}

Si les antidépresseurs sont utilisés par les sportifs en vue d'améliorer leurs performances, ces médicaments font l'objet d'un détournement d'usage également chez les non sportifs. Alors que certaines substances ne sont détournées de leur usage médical que par des sportifs (stéroïdes anabolisants, corticoïdes, EPO...), les AD connaissent les faveurs de tous. Paradoxalement l'on dispose de moins de données sur les abus d'usage chez les sportifs, pour lesquels des contrôles sont organisés... que chez les cadres. Comme les sportifs, les cadres aussi sont astreints à la performance et les nouvelles techniques de management incitent réellement nombre d'entre eux au dopage. Cette situation devient un véritable fléau qui progresse à tous les échelons dans les entreprises. On admet aujourd'hui qu'un cadre sur cinq est dopé, ce phénomène se propage à d'autres catégories, il y a donc lieu de prendre très rapidement un certain nombre de mesures. Parmi elles, il conviendrait d'interdire les médicaments à l'origine de syndromes de sevrage ou de pharmacodépendance : parmi les nouveaux $\mathrm{AD}, 3$ sur 6 encore commercialisés présentent cette caractéristique chez plus de $13 \%$ des sujets traités (4). La prescription des AD doit également être mieux encadrée, mieux contrôlée. Enfin, comme le dit si bien le Pr Zarifian il faut aussi lutter contre la "médicalisation de l'existentiel" et s'attacher aux causes du mal plutôt qu'à ses effets, il existe des alternatives à la camisole chimique : de l'accompagnement à la psychothérapie (42).

Tableau III : Antidépresseurs retirés du marché ou non commercialisés.

\begin{tabular}{|c|c|c|c|c|}
\hline Substance & Spécialité & Toxicité & $\begin{array}{l}\text { Date de mise } \\
\text { sur le marché }\end{array}$ & $\begin{array}{c}\text { Date de } \\
\text { retrait }\end{array}$ \\
\hline Zimélidine & - & Guillain Barré & $\begin{array}{c}\text { Non } \\
\text { commercialisê } \\
\text { en France }\end{array}$ & 1983 \\
\hline Nomifensine & Alival $\mathbb{B}$ & $\begin{array}{c}\text { Anémie } \\
\text { hémolytique }\end{array}$ & 1977 & 1987 \\
\hline Indalpine & Upsène® & Agranulocytose & 1983 & 1987 \\
\hline Métapramine & Timaxel® & $\begin{array}{c}\text { Fièvre } \\
\text { médicamenteuse }\end{array}$ & 1985 & 1992 \\
\hline Minaprine & Cantor® 100 & $\begin{array}{c}\text { Toxicité } \\
\text { neurologique }\end{array}$ & 1990 & 1995 \\
\hline Minaprine & Cantor ${ }^{\circledR 50}$ & $\begin{array}{c}\text { Toxicité } \\
\text { neurologique }\end{array}$ & 1995 & 1996 \\
\hline Amineptine & Survector (1) & Surconsommation & 1976 & $\begin{array}{c}\text { Janvier } 1999 \\
\text { Juin } 1999\end{array}$ \\
\hline
\end{tabular}




\section{Références}

1. Bruno O. Les extases olympiques de l'ex stasi. Science et Vie. $1999 ; 206: 88-93$.

2. Gava M.J. Vos collaborateurs se dopent-ils ? L'essentiel du management 1998, novembre : 104-108.

3. Tracqui A., Ludes B. Médicaments psychotropes. in : Kintz P., Elsevier, ed, Toxicologie et Pharmacologie médicolégales. $1998: 191$.

4. Blayac J.P., Hillaire-Buys D., Peyrière H. La pharmacovigilance des nouveaux antidépresseurs : évaluation des troubles neuro-psychocomportementaux. Thérapie. 1997 ; $52: 117-122$.

5. Raymond-Schwartzmann V. Antidépresseurs et pharmacodépendance. Toxicorama. $1998 ; 10$ (4) : 217-223.

6. Nores J.M., Toro P., Rambaud S. Toxicomanie à la clomipramine responsable d'un bloc de branche droit résolutif à l'arrêt de l'intoxication. Thérapie. 1998 ; 43 : 501-502.

7. Randy Eicher E. Ergolytic drugs in medicine and sports. Am. J. Med. 1993 ; 94 : 205-211.

8. Lejoyeux M. Dépendance aux antidépresseurs : authentique addiction ? Encéphale $1995 ; \mathrm{Sp} \mathrm{I}:$ 63-65.

9. Castot A., Benzaken C., Wagniart F., Efthymiou M.-L. Surconsommation d'amineptine : analyse de 155 cas, bilan de l'enquête officielle coopérative des Centres Régionaux de Pharmacovigilance. Thérapie. $1990 ; 45: 399-405$.

10. Farella V., Sberna F., Knöpfel B. et al. Acne-like eruption caused by amineptine. Int. j. Dermatol. 1996 ; 35 : 892-893.

11. Beylot-Barry M., Boisseau-Garsaud A.-M., Ly S. et al. Montrous acne due to amineptine. Four observations. Eur. J. Dermatol. $1996 ; 6: 97-100$.

12. Journées Françaises 1994 de Pharmacovigilance. Revue Prescrire. $1995 ; 15$ (149) : 190.

13. Ayotte C., Goudreault D. The detection of new synthetic drugs in athlete's urine samples. J. Toxicol. Toxin Rewiews. 1999 ; 18 (2) : 113-123.

14. Journal Officiel du 26 mars 1994 : page 4578.

15. Arrêtés des 30/1/99 (délivrance hospitalière uniquement) et 30/6/99 (retrait total). Non publiés au J.O.

16. Kintz P. Amineptine abuse detected by hair analysis. Toxichem + Krimtech. $1994 ; 61: 70-73$.

17. Chagraoui A., Vasse M., Protais P. Interaction of amineptine with agents modifyinf dopaminergic transmission. Clin. Neuropharmacol. 1989; 12 (Sp 2) : S19-31.

18. Chagraoui A., Vasse M., Protais P. Effects of chronic treatments with amineptine and desipramine on motor responses involving dopaminergic systems. Psychopharmacology (Berl). $1990 ; 102$ (2) : 201-206.

19. Mercuri N.B., Calabresi P., Bernardi G. The electrophysiological actions of dopamine and dopaminergic drugs on neurons of the substantia nigra pars compacta and ventral tegmental area. Life Sci. $1992 ; 51$ (10) : 711-718.

20. Bruhwyler J., Chleide E., Rettori M.C., Poignant J.C., Mercier M. Amineptine improves the performance of dogs in a complex temporal regulation schedule. Pharmacol. Biochem Behav. 1993 ; 45 (4) : 897-903.

21. Garattini S. Pharmacology of amineptine, an antidepressant agent acting on the dopaminergic system : a review. Int. Clin. Psychopharmacol. 1997 ; 12 (Sp.3) : S15-9.
22. Caccia S. Metabolism of the newer antidepressants. An overview of the pharmacological and pharmacokinetic implications. Clin. Pharmacokinet. 1998 ; 34 (4) : 281302.

23. Lambert H., Société de Toxicologie. Paris, 10.1.1994.

24. Breggin P.R. Bibliographie "dopage et drogues de la performance ". Talking Back to Prozac : What Doctors won't tell you about todday's most controversial drug. 1995 ; St Martin Mass Market Paper.

25. Tracqui A., Ludes B. Médicaments psychotropes. in : Kintz P., Elsevier, ed, Toxicologie et Pharmacologie médicolégales. $1998: 233-274$.

26. Bourguignon P. Dangers of fluoxétine. Lancet 1997 ; $349: 214$.

27. Ménecier P., Ménecier-Ossia L., Bern P. Dépendance et tolérance à la fluoxétine. A propos d'une observation. Encéphale $1997 ; 23: 400-401$.

28. Pagliaro L.A., Pagliaro A.M. Fluoxetine abuse by an intravenous drug user. Am. J. Psychiatry. $1993 ; 150$ (2) : 1898.

29. Lazowich A.L., Levin G.M. Potential withdrawal syndrome associated with SSRI discontinuation. Ann. Pharmacother. $1995 ; 29: 1284-1285$.

30. www.socialaudit.org.uk

31. Rimane M. Malades ou dopés ? Science et Vie 1999, 206, 74-78.

32. Neuvonen P.J., Pohjota-Sintonen S., Tacka U. et al. Five fatal cases of serotonin syndromes after moclobemidecitalopram or moclobemide-clomipramine overdoses. Lancet. 1993 ; $342: 1419$.

33. Louie A.K., Lannon R.A., Ajari L.J. Withdrawal reaction after sertraline discontinuation. Am. J. Psychiatry 1994 ; 151 (3) : 450-451.

34. Debattista C., Schatzberg A.F. Physical symtoms associated with paroxetine withdrawal. Am. J. Psychiatry. 1995 ; 152 (8) : 1235-1236.

35. Barr L.C., Goodman W.K., Price L.H. Physical symptoms associated with paroxetine discontinuation. Am. J. Psychiatry. $1994 ; 151$ (2) : 289.

36. Kudrin V.S., Sergeeva S.A., Krasnykh L.M., Miroshnichenko I.I., Grekhova T.V., Gainetdinov R.R. The effect of bromantane on the dopamin- and serotoninergic systems of the rat brain. Eksp. Klin. Farmakol. 1995 ; 58(4) : 8-11.

37. Morozov I.S., Kleimenova N.N. The effect of bromantane on the physical work capacity of laboratory animals. Eksp. Klin. Farmakol. 1998 ; 61(6) : 51-3.

38. Morozov I.S., Klimova N.V., Karpova T.D., Shestopalov S.S. The characteristics of the neuropsychotropic activity of bromantane in laboratory animals. Eksp. Klin. Farmakol. 1999 ; 62(2) : 3-6.

39. Seredenin S.B., Miramedova A.G., Kozlovskaia M.M. The effect of bromantane on the behavior of inbred mouse strains with different phenotypes of emotional stress reaction. Eksp. Klin. Farmakol. 1999 ; 62(3) : 3-6.

40. Bugaeva L.I., Spasov A.A., Morozov I.S. The effect of bromantane on the erythro-and leukocytic profile of the peripheral blood in rats. Eksp. Klin. Farmakol. 1999 ; $62(4): 40-3$.

41. Burnat P., Bromantan, a new doping agent. Lancet. 1997 ; 350 (9082) : 963-4.

42. Zarifian E. Le prix du bien-être. Odile Jacob ed. 1996 